\title{
Valoración económica de ensilajes de Zea mays y forrajes como una alternativa de nutrición de bovinos en la altillanura
}

\section{Economic evaluation of Zea mays silage and fodder as alternative nutrition of cattle in high plains}

\author{
Beltrán Polania Diego Alejandro² y Lemus Alarcón Lázaro Hugo ${ }^{1}$ \\ ${ }^{1}$ I.A. MSc. Universidad de los Llanos y \\ ${ }^{2}$ MVZ. MSc. Docente Universidad de los Llanos \\ dimartin40@yahoo.com
}

Recibido 02 de Octubre 2014, Aceptado 10 de Abril 2015

\section{RESUMEN}

El trabajo se realizó en una finca ganadera ubicada en la vereda La Guardiana del Municipio San Martín de los Llanos en un paisaje de Altillanura con suelos clase III, con el objetivo determinar la opción económica de mayor viabilidad en la producción de ensilaje para la alimentación de bovinos en parcelas, donde se establecieron los siguientes tratamientos: Zea mays y pasto de corte Pennisetum purpureum en monocultivo y asociados con la leguminosa Mucuna deeringiana (Bort) Merr. El área de estudio tiene $350 \mathrm{msnm}$, temperatura y precipitación media de $24^{\circ} \mathrm{C}$ y $3200 \mathrm{~mm}$, allí se establecieron los tratamientos con un diseño experimental en un bloque al azar subdividido en 4 parcelas para cada uno con cuatro repeticiones, para un total de 16 parcelas, con un área de $5.000 \mathrm{~m}^{2}$, siendo la totalidad del experimento de $80.000 \mathrm{~m}^{2}$. Se tomaron manualmente 20 muestras del forraje fresco en $1 \mathrm{~m}^{2}$ de cada parcela, las cuales fueron pesadas y ensiladas para calcular rendimiento del forraje fresco y ensilado por hectárea. Las parcelas se cultivaron, cosecharon y ensilaron calculando su costo por hectárea, con esta información y el costo comercial se realizó el análisis económico. El rendimiento de biomasa en forraje fresco, presentó el mejor resultado en el tratamiento que involucró al Zea mays con Mucuna deeringiana (Bort) Merr siendo de 66.110 $\mathrm{kg} / \mathrm{ha}$, seguido del Zea mays con $65.406 \mathrm{~kg} / \mathrm{ha}$, siendo el más bajo Pennisetum purpureum en monocultivo con $30.434 \mathrm{~kg} / \mathrm{ha}$. La variable rendimiento de biomasa 
en forraje ensilado presentó su mayor rendimiento en asociación de Zea mays con Mucuna deeringiana (Bort) Merr con $(61.181 \mathrm{~kg} / \mathrm{ha})$, seguido de Zea mays con (60.649 kg/ha) y Pennisetum purpureum con $30.000 \mathrm{~kg} / \mathrm{ha}$. Se demostró en el análisis económico que la mayor rentabilidad se obtuvo ensilando Zea mays en asociación con Mucuna deeringiana (Bort) Merr, generando COP\$6.931.807 por hectárea de biomasa ensilada, seguido de Zea mays en monocultivo con COP\$6.871.532 siendo la más baja la de Pennisetum purpureum en monocultivo COP\$3.399.000.

Palabras clave: Cultivos forrajeros, Pennisetum purpureum, maíz, Mucuna deeringiana.

\section{ABSTRACT}

The work was done on a cattle farm in the village of La Guardiana of the municipality of San Martin de los Llanos in scenic class III floors high plains, in order to determine the most viable economic option in the production of silage for feeding cattle on plots where the following treatments were established: Zea mays and cutting grass Pennisetum purpureum in monoculture and associated with the legume Mucuna deeringiana (Bort) Merr). The study area is 350 meters above sea level, temperature and average precipitation of $24^{\circ} \mathrm{C}$ and $3.200 \mathrm{~mm}$, there treatments with an experimental design were established in randomized block into 4 plots each with four replicates for a total of 16 plots, with an area of $5.000 \mathrm{~m}^{2}$, with the entire experiment than $80.000 \mathrm{~m}^{2} .20$ samples of fresh forage in $1 \mathrm{~m}^{2}$ of each plot are manually taken; which were weighed to calculate performance and ensiled fresh forage and silage per hectare. The plots were grown, harvested and ensiled calculating the cost per hectare, with this information and the commercial cost economic analysis. The biomass yield in fresh fodder, showed the best results in the treatment that involved Zea mays with Mucuna deeringiana (Bort) Merr being of $66.110 \mathrm{~kg} / \mathrm{ha}$, followed by Zea mays with $65.406 \mathrm{~kg} / \mathrm{ha}$, being the lowest Pennisetum purpureum in monoculture with $30.434 \mathrm{~kg} / \mathrm{ha}$. The variable biomass yield in silage showed the highest yield in association with Mucuna deeringiana (Bort) Merr with Zea mays $(61.181 \mathrm{~kg} / \mathrm{ha})$, followed by Zea mays with $(60.649$ 
$\mathrm{kg} / \mathrm{ha}$ ) and Pennisetum purpureum $30.000 \mathrm{~kg} / \mathrm{ha}$. It was shown in the economic analysis that most performance was achieved ensiling Zea mays in partnership with Mucuna deeringiana (Bort) Merr, generating COP\$6.931.807 per hectare of ensiled biomass, followed by Zea mays in monoculture with COP $\$ 6.871 .532$ being the lowest Pennisetum purpureum in monoculture COP\$3.399.000.

Keywords: Forage crops, Pennisetum purpureum, corn, Mucuna deeringiana.

\section{RESUMO}

O trabalho foi feito em uma fazenda de gado na aldeia de La Guardiana do município de San Martin de los Llanos em uma paisagem de planaltos com solos de classe III, a fim de determinar a opção econômica mais viável na produção de silagem para alimentação gado em parcelas onde foram estabelecidos os seguintes tratamentos: Zea mays e de cortar relva Pennisetum purpureum em monocultura e associado com a leguminosa Mucuna deeringiana (Bort) Merr). A área de estudo está a 350 metros acima do nível do mar, temperatura e precipitação de $24^{\circ} \mathrm{C}$ e $3.200 \mathrm{~mm}$, existem tratamentos com um design experimental foram estabelecidos em blocos ao acaso, em 4 parcelas, cada uma com quatro repetições, num total de 16 parcelas, com uma área de $5.000 \mathrm{~m}^{2}$, com toda a experiência de $80.000 \mathrm{~m}^{2} .20$ amostras de forragem fresca em $1 \mathrm{~m}^{2}$ de cada parcela é tomado manualmente; que foram pesados para calcular o desempenho e forragem ensilada fresco e silagem por hectare. As parcelas foram cultivado, colhido e ensilado calcular o custo por hectare, com esta informação e análise econômica de custo comercial. A produção de biomassa de forragem fresca, apresentou os melhores resultados no tratamento envolvendo a Zea mays Mucuna deeringiana (Bort) Merr sendo de $66.110 \mathrm{~kg} / \mathrm{ha}$, seguido de Zea mays com $65.406 \mathrm{~kg} / \mathrm{ha}$, sendo o menor Pennisetum purpureum em monocultivo com $30.434 \mathrm{~kg} / \mathrm{ha}$. A produção de biomassa variável na silagem apresentou o maior rendimento no tratamento que envolveu Zea mays associação com Mucuna deeringiana (Bort) Merr com (61.181 kg/ha), seguido de Zea mays com (60.649 kg/ha) e Pennisetum purpureum 30.000 kg/ha. Mostrou-se na análise econômica que mais desempenho foi alcançado ensilagem Zea mays em parceria com 
Mucuna deeringiana (Bort) Merr, gerando COP\$6.931.807 por hectare de biomassa ensilado, seguido de Zea mays em monocultura com COP\$6.871.532 sendo o mais baixo Pennisetum purpureum em monocultura COP\$3.399.000.

Palavras-chave: Culturas forrageiras, Pennisetum purpureum, milho, Mucuna deeringiana.

\section{INTRODUCCIÓN}

La altillanura colombiana está formada por dos paisajes con características muy definidas: la disectada (serranía), y la plana. Su vegetación está compuesta por arbustivas y gramíneas nativas, siendo estas últimas las de mayor proporción de la Altillanura disectada, debido a su adaptación en condiciones de: clima con un periodo de sequía comprendido entre Noviembre y Abril, y suelos de baja fertilidad que se caracterizan por su alto contenido de aluminio, reducido nivel de bases intercambiables (calcio y magnesio) y poco contenido de materia orgánica; por lo tanto, estos paisajes han permitido el desarrollo de la ganadería extensiva, porque la producción de forraje es limitada afectando la producción de carne y leche (Jamioy, 2011).

La ganadería extensiva se ha mantenido por más de cien años en estas condiciones, sin embargo algunos ganaderos emprendedores introdujeron mejoría genética en el píe de cría mediante la adaptación de padrotes de la raza cebú, creando mestizaje con las razas Europeas que habían traído los Españoles durante la conquista y la colonia, convirtiendo el hato en una ganadería cebú al cabo de unos años. Posteriormente, mediante programas de inseminación, introdujeron razas lecheras como Simmenthal, Pardo Suizo, Holstein y otras, obteniendo un mestizaje conocido como doble propósito, que exige adecuados requerimientos nutricionales para producir leche y carne (CORPOICA, 2003); puesto que las praderas nativas no tienen las condiciones para proporcionarlos en lo relacionado a cantidad y calidad de forraje. Poco a poco los ganaderos introdujeron especies gramíneas como Brachiaria decumbens y B. humidicola, 
llegando al punto en el que hoy en día, la mayoría de las praderas están cultivadas con estas dos especies.

A pesar de esta mejora en el forraje para el ganado bovino, no ha sido suficiente cuando se requiere alimentar durante la temporada de sequía, porque los pastos se secan y los animales reducen drásticamente la producción de leche y carne, perdiendo entre 10 y $15 \%$ de su peso corporal, frente a esta situación los ganaderos han buscado alternativas suplementando sus animales con concentrado, pasto de corte fresco o con ensilaje, siendo las limitantes de las primeras alternativas: el alto costo del concentrado, y la ausencia de agua para regar en verano y mantener fresco el cultivo de pasto de corte; aunque el ensilaje es más costoso con relación a producir pasto de corte fresco, esta opción es más económica que alimentar el ganado con concentrado, además el ensilaje ofrece la garantía de conocer la cantidad de alimento de reserva con el cuál se dispone (Cuartas et al., 2009).

Para solucionar la escasez de forraje durante la época de sequía, muchos ganaderos han cultivado maíz y pasto de corte para ensilar o para ofrecerlo fresco, sin embargo la solución es encontrar la alternativa más rentable para cada explotación ganadera, con este objetivo se han establecido cultivos asociados entre diferentes gramíneas y leguminosas forrajeras; con el fin de encontrar las especies más adecuadas para cada finca, que asociadas desarrollan sinergismo para producir la mayor cantidad de forraje de buena calidad (CORPOICA, 2003).

En este sentido Chacón y Vargas, (2009) determinaron la digestibilidad y calidad del Pennisetum purpureum a tres edades de rebrote $(60,75$ y 90 días) encontrando un contenido de materia seca de $13.03-14.43 \%$, proteína cruda 8.42 9.56\%, extracto etéreo $1.29-1.41 \%$, cenizas $13.61-14.47 \%$ fibra detergente neutro $73.78-76.91 \%$ y fibra detergente acido $46.53-51.83 \%$. Así mismo, Zambrano et al., (2006) determinaron un contenido de materia seca de $20 \%$, proteína cruda $9.49 \%$, extracto etéreo $2.47 \%$, fibra cruda $35 \%$, ceniza $8 \%$ y extracto libre de nitrógeno $45.04 \%$. 
El ensilado de cultivos forrajeros o de subproductos industriales podría ser una contribución importante para optimizar el funcionamiento de los sistemas de producción animal en zonas tropicales y subtropicales, pero su empleo es todavía muy escaso (Garcés et al., 2004). El ensilaje de maíz se destina comúnmente al consumo del ganado vacuno de carne o leche, donde el valor nutricional del forraje cosechado está determinado principalmente por la tasa de producción animal que éste forraje genera, es decir por la eficiencia en la conversión del alimento en producto animal (carne o leche). La conversión está afectada por la digestibilidad del forraje, la cantidad consumida y la eficiencia en su utilización, estos factores están interrelacionados y todos a su vez, condicionados por las características del animal y de la planta (Bertoia, 2010). Castillo et al., (2009) determinaron el valor nutricional del ensilaje de maíz a los 60 días de maduración, encontrando materia seca $19.76-23.60 \%$, proteína cruda $8.88-11.36 \%$, fibra detergente neutro $50.10-61.26 \%$, fibra detergente acida $28.52-36.52 \%$, extracto etéreo $1.39-1.98 \%$, cenizas $5.68-7.49 \%$ y digestibilidad in vitro de la materia seca $69.89-79.78 \%$.

La asociación de Zea mays (maíz) y Pennisetum purpureum (pasto elefante) con Mucuna deeringiana (Bort) Merr (mucuna) monocultivo de maíz y elefante son una de las posibles asociaciones gramínea con leguminosa y de los monocultivos de gramíneas que han ensayado algunos ganaderos. Con este trabajo se pretende hacer un aporte para que los ganaderos de la Altillanura colombiana tengan una opción más y considerando los recursos que se encuentran en su hato, analicen si alguna le es favorable en términos de rendimiento económico.

\section{METODOLOGÍA}

\section{Condiciones de la zona}

El trabajo experimental se realizó en la finca Santa Marta, que se halla ubicada en la vereda La Guardiana, en el municipio de San Martín de los Llanos, del departamento del Meta, siendo sus condiciones climatológicas y edafológicas: 350 msnm temperatura y precipitación anual media de $24^{\circ} \mathrm{C}$ y $3.200 \mathrm{~mm}$, se encuentra 
en un paisaje de altillanura plana, caracterizada por su suelo que se halla agrupado en la clase III (Alcaldía de San Martin, 2012). La hacienda se halla a 18 $\mathrm{Km}$ de la cabecera municipal, tiene una extensión de 730 hectáreas y de ella se destinaron ocho hectáreas para realizar el cultivo del experimento.

\section{Distribución del experimento}

Se planificó el diseño experimental en bloques completamente al azar, subdividido en cuatro (4) parcelas para cada uno de los tratamientos, con cuatro (4) repeticiones y distribuidos totalmente al azar, asignando a cada lote una combinación que permitió realizar comparaciones en iguales condiciones de agrupaciones, separación, densidad de cultivo y procedimiento de ensilaje; se repitieron los tratamientos con el objeto de dar mayor confiabilidad a la muestra del experimento, bajo los mismos parámetros de los bloques anteriores.

Se adoptaron siembras de monocultivo de maíz y en asociación con mucuna, monocultivo de elefante y asociado con esta leguminosa, para cada tratamiento se asignó un área de dos (2) hectáreas de tal manera que la totalidad del experimento fue de 80.000 metros cuadrados (Figuras 1 y 2 ).
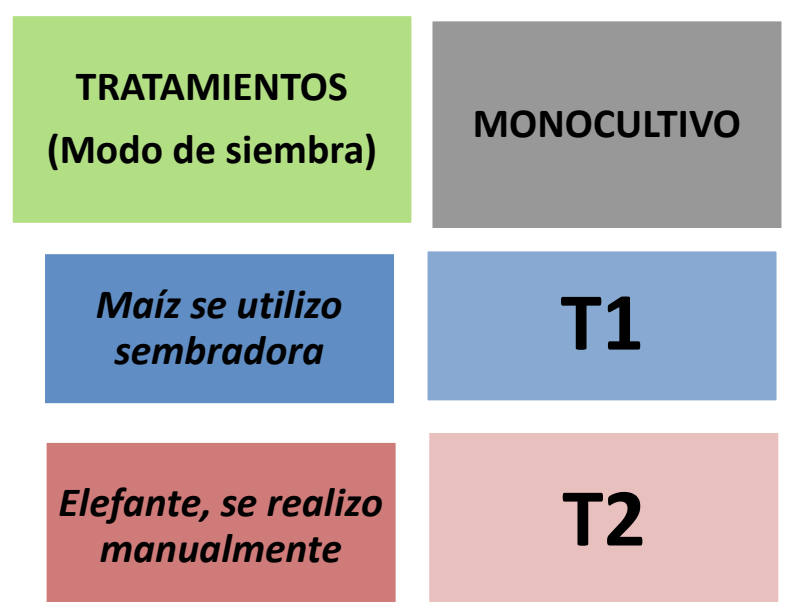

Tabla 1. Conformación de cada bloque por tratamiento
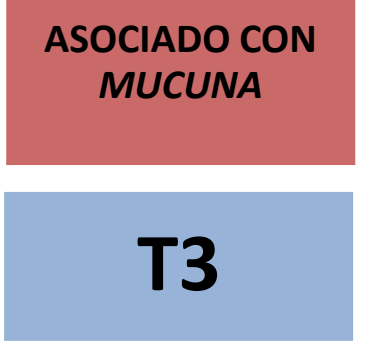

T4 


\begin{tabular}{|c|c|c|c|c|c|c|c|}
\hline \multicolumn{2}{|c|}{ PARCELA 1 } & \multicolumn{2}{c|}{ PARCELA 2 } & \multicolumn{2}{c|}{ PARCELA } & \multicolumn{2}{c|}{ PARCELA 4 } \\
\hline Bloque 1 & Bloque 2 & Bloque 1 & Bloque 2 & Bloque 1 & Bloque 2 & Bloque 1 & Bloque 2 \\
\hline$T_{1}$ & $T_{3}$ & $T_{1}$ & $T_{3}$ & $T_{1}$ & $T_{3}$ & $T_{1}$ & $T_{3}$ \\
\hline$T_{2}$ & $T_{4}$ & $T_{2}$ & $T_{4}$ & $T_{2}$ & $T_{4}$ & $T_{2}$ & $T_{4}$ \\
\hline
\end{tabular}

Figura 2. Distribución de los tratamientos en el terreno

\section{Diseño estadístico}

El diseño estadístico que se aplicó en este experimento fue bloques completamente al azar con cuatro tratamientos, para un total de 16 unidades experimentales, donde el modelo matemático fue:

$$
\gamma=\mu+\mathrm{T} i+\beta j+\mathrm{Eij}
$$

- $\gamma i j$ : Las variables aleatorias que representa la observación (i)-ésima del bloque (j)-ésimo, que en este experimento fueron: rendimiento de biomasa en forraje fresco, en forraje ensilado y evaluación económica (costos totales, unitarios y beneficio).

- $\mu$ : es un efecto constante, media global.

- Ti: El efecto producido por el nivel i-ésimo del factor principal, Parcelas: 1 (monocultivo maíz), 2 (monocultivo de elefante), 3 (asociación maíz con mucuna) y 4 (asociación elefante con mucuna).

- $\beta j$ : El efecto producido por el nivel j-ésimo del factor secundario o factor de bloques: 1 (monocultivo) y 2 (asociación).

- Eij: error experimental.

\section{Consideraciones de la siembra}

Maíz monocultivo: Se eligió una semilla híbrida que tiene grano amarillo, presenta una altura de plantas de 2.7 metros y es tolerante a las enfermedades tropicales, su edad para cosecharlo en estado de grano pastoso esta entre 80 y 90 días 
después de la germinación, dependiendo de las condiciones climáticas. Se estableció colocando 10 semillas por metro lineal, y distanciado los surcos a 80 cm, para alcanzar una población de ciento veinticinco mil (125.000) plantas por hectáreas. Se sembraron $23.7 \mathrm{~kg}$ de semilla por cada parcela, lo que requirió 94.8 $\mathrm{kg}$ para las cuatro áreas sembradas, se utilizó sembradora, dejando las semillas cubiertas con una capa de suelo de $2 \mathrm{~cm}$.

Maíz asociado con mucuna: Se eligió esta leguminosa por su rusticidad, vigor y gran capacidad de crecimiento, para que compita con la gramínea. La semilla de mucuna se sembró manualmente después de la siembra del maíz, a una distancia de $125 \mathrm{~cm}$ sobre el surco de maíz, con esta distancia se estableció una densidad de 10.000 plantas de mucuna por hectárea, la cantidad de semilla utilizada fue 3.6 $\mathrm{kg}$ por parcela, por ello para la asociación Zea mays se necesitó $14.4 \mathrm{~kg}$. En cuanto a esta asociación el maíz se sembró en la misma densidad del monocultivo, por lo tanto la cantidad de semilla empleada fue $94.8 \mathrm{~kg}$ para este tratamiento.

Elefante asociado con mucuna: El pasto se sembró con el mismo sistema y en la misma cantidad de semillas utilizadas en el tratamiento de monocultivo, por lo tanto, se requirieron $5.600 \mathrm{~kg}$ de estacas. La mucuna se estableció buscando sembrar la misma densidad de plantas de la leguminosa que se empleó en la asociación maíz (3.6 kg por parcela), para ello se hizo siembra alternando en los surcos de elefante un surco de mucuna. La cantidad total de semilla empleada en el experimento fue de $189.6 \mathrm{~kg}$ de Zea mays, $28.8 \mathrm{~kg}$ de mucuna y $11.2 \mathrm{~kg}$ de estacas de elefante.

La fertilización realizada según análisis de suelos, en cantidades por hectárea: 1.5 toneladas de cal dolomítica, que se aplicó durante la preparación del terreno; 150 $\mathrm{kg}$ de difosfato de amonio, el cual se aplicó la totalidad al momento de la siembra, $150 \mathrm{~kg}$ de cloruro de potasio, repartido $30 \%$ al momento de la siembra, $40 \%$ a los 30 día después de la siembra y $30 \%$ a los 45 días después de la siembra; $120 \mathrm{~kg}$ de urea; aplicándose $40 \%$ a los 30 días después de la siembra y $60 \%$ a los 45 
días después de la siembra, $20 \mathrm{~kg}$ de borozinco, el 50\% al momento de la siembra y el $50 \%$ a los 30 días después de la siembra.

En el desarrollo del cultivo se realizó raleo del maíz a los 10 días de sembrado en los tratamientos que lo involucraban, la actividad consistió en eliminar desde 1 a 3 plantas de maíz por metro lineal, deshierba a los 15 días de sembrado, y 10 días después se realizó el aporque con tractor, no se emplearon herbicidas pre o post emergentes puesto que en la asociación gramínea-leguminosa puede afectarse el desarrollo de esta última; para el control de insectos, dos días después de la siembra se hizo una aplicación de carbofuran: 2,3-dihidro-2,2-dimetilbenzofuran-7il-metilcarbamato líquido sobre el surco en una dosis de $250 \mathrm{~cm}^{3}$ por hectárea en 300 litros de mezcla, con el fin de evitar daños por tierreros-trozadores presentes en el suelo, una vez hecho el aporque se hizo la primera liberación de Trichogramma spp en dosis de 25 pulgadas por hectáreas, luego semanalmente se hicieron otras tres liberaciones en igual concentración, para prevenir el daño de Diatrea Saccharallis (pasador) y Spodoptera frugiperda (gusano cogollero), a los 60 días después de la siembra se presentó un ataque de Spodoptera, lo cual se controló aplicando de manera localizada Dipel (Bacillus thurigiensis) en dosis de 250 gramos por hectárea; la fertilización se hizo manual y al voleo en las fechas establecidas a los 30 y 45 días después de la siembra, y a los 80 días se inició la recolección del cultivo, sin presentarse enfermedades, evidenciando un estado sanitario óptimo.

\section{Proceso de producción de ensilaje}

La cosechadora de forraje pica el forraje y lo lanza por una canal a un remolque, cuando este se llena, los operarios lo desenganchan y en el tractor que lleva la cosechadora se monta un remolque vacío, mientras que el lleno lo transporta otro tractor, y es llevado a una báscula electrónica, allí se hace el pesaje donde previamente se había pesado vacío cada remolque, posteriormente lo lleva al sitio del silo, donde se descarga y se distribuye, y por cada dos toneladas se aplicó 1.0 kg de melaza disuelta en agua en proporción 1:1 y con el mismo tractor se hizo la 
compactación del forraje picado, cada vez que la cosechadora llena un remolque se repite la operación hasta cosechar cada parcela.

El forraje de cada parcela fue ensilado independientemente para conocer el costo de ensilado por kg en cada tratamiento, el ensilaje se cubrió con un plástico negro, y se prensó por los bordes con una capa de tierra de $10 \mathrm{~cm}$ de gruesa y con un ancho de $60 \mathrm{~cm}$, en el extremo donde se terminó el sellado se colocó un tubo PVC perforado en un extremo con agujeros de $0.3 \mathrm{~cm}$ de diámetro, conectando a una aspiradora para extraer el oxígeno, puesto que las bacterias que fermentan el forraje son anaeróbicas, luego se retiró el tubo PVC y se acabó de sellar el silo de montón con tierra.

Se estableció un ensilaje experimental para determinar el rendimiento de cada uno de los tratamientos, para ello se cosechó manualmente cortando las plantas a una altura de $10 \mathrm{~cm}$ del suelo, se hizo el pesaje del forraje fresco en la balanza de precisión, se llevaron las plantas hasta la pica-pasto y se empezó el llenado de la bolsa de plástico negro (calibre 7) la cual media $90 \mathrm{~cm}$ de largo y $60 \mathrm{~cm}$ de ancho, en ella se compactó el forraje picado con un "pizon" y se introdujo un tubo PVC de media pulgada con perforaciones de 0.3 milímetros de diámetro cerca de un extremo, introduciéndolo en la bolsa y en otro extremo (externo) del tubo PVC, se conectó una aspiradora, para extraer el oxígeno, luego se retiró el tubo PVC y se aseguró el plástico, quedando totalmente sellada la bolsa plástica, posteriormente la bolsa plástica llena se introdujo en una bolsa de polipropileno, para protegerla de rompimientos o dilataciones.

\section{Procedimiento de recolección de muestras}

Se tuvo especial cuidado en la toma de los registros básicos para el análisis del rendimiento, haciendo un muestreo altamente representativo, teniendo en cuenta la totalidad del terreno y considerando que el tamaño de la muestra fuera de un $\mathrm{N}$ $=20$ por tratamiento (Carranza, 1994). Cada una de las muestras se tomó completamente al azar, cosechando en el surco el equivalente de plantas correspondiente a $1 \mathrm{~m}^{2}$ en maíz en monocultivo, asociado con mucuna $1.25 \mathrm{~m}$ 
lineales; y con elefante en monocultivo así como asociado $2.0 \mathrm{~m}$ lineales. Las plantas fueron cortadas a $10 \mathrm{~cm}$ de la superficie del suelo, luego colocadas en bolsa plástica y pesadas en la balanza, posteriormente cortadas en la pica-pasto y por último fueron ensiladas en bolsas de manera independiente. Para los tratamientos que involucraron elefante la determinación del rendimiento en forraje fresco se hizo un día antes de la cosecha, es decir el día 79 después de la siembra al maíz.

\section{Cálculo de costos}

Esta contabilidad para cada uno de los tratamientos se realizó bajo el sistema de costos totales ya que se tiene en cuenta todos los gastos que inciden en el producto. Para la ejecución del trabajo realizado en un tiempo de 6 meses, en un área de $80.000 \mathrm{~m}^{2}$, se clasificaron los costos en directos (preparación del terreno, semillas, correctivos, fertilizantes, insecticidas, herbicidas, fungicidas y mano de obra) e indirectos (asistencia técnica, administración, arrendamiento de terreno, interés al capital e imprevistos). Inicialmente se estimó los costos de producción de forraje fresco por $\mathrm{kg}$ y luego se calculó el costo total de elaboración de forraje ensilado por kg, el cual se le adicionó al costo de producción de forraje fresco.

\section{RESULTADOS Y DISCUSIÓN}

\section{Composición nutricional de las especies evaluadas}

En la Tabla 1 se presenta la composición nutricional del maíz, mucuna y elefante utilizados en el experimento, obtenidos en el Laboratorio de Nutrición Animal de la Universidad de los Llanos.

\section{Rendimiento de forraje fresco y ensilado}

El rendimiento por tratamiento y por hectárea fue el resultado de extrapolar los datos de las muestras para cada tratamiento (Tabla 2), según los pesos promedios obtenidos del modelo estadístico y considerando las siguientes estimaciones: fueron 16 parcelas con un área de $5.000 \mathrm{~m}^{2}$ cada una, para un área total del cultivo de $80.000 \mathrm{~m}^{2}$, con una densidad de siembra de 100.000 plantas por 
hectárea, es decir 50.000 plantas en cada parcela, para un total de 800.000 plantas en el cultivo, utilizando un área de cada muestreo de $1 \mathrm{~m}^{2}$, analizándose 80 muestras por tratamiento.

Tabla 1. Composición nutricional de las especies evaluadas

\begin{tabular}{cccc}
\hline $\begin{array}{c}\text { Fracción } \\
\text { Nutricional (\%) }\end{array}$ & Zea mays & $\begin{array}{c}\text { Mucuna } \\
\text { deeringiana } \\
\text { (Bort) Merr }\end{array}$ & $\begin{array}{c}\text { Pennisetum } \\
\text { purpureum }\end{array}$ \\
\hline Materia Seca & 40.81 & 15.2 & 16.9 \\
Proteína & 7.6 & 22.15 & 9.2 \\
Extracto etéreo & 2.5 & 8.7 & 1.34 \\
Fibra detergente & 10.79 & 64.77 & 63.72 \\
$\quad$ neutro & & 48.95 & 46.36 \\
Fibra detergente & 2.68 & 3.4 & 13.79 \\
ácido & 9.11 & 5.7 & 30.17 \\
Cenizas & 21.51 & & \\
Fibra Cruda & & & \\
\hline
\end{tabular}

Tabla 2. Rendimiento de forraje fresco tratamiento por hectárea

\begin{tabular}{ccccc}
\hline Tratamientos & $\mathbf{T}_{\mathbf{1}}$ & $\mathbf{T}_{\mathbf{2}}$ & $\mathbf{T}_{\mathbf{3}}$ & $\mathbf{T}_{\mathbf{4}}$ \\
\hline Tamaño de cada muestra $\left(\mathrm{m}^{2}\right)$ & 1.0 & 1.0 & 1.0 & 1.0 \\
Número de muestras por & 80 & 80 & 80 & 80 \\
tratamiento & & & & \\
Peso totalidad de muestras $(\mathrm{kg})$ & $523.4^{\mathrm{a}}$ & $243.4^{\mathrm{b}}$ & $528.8^{\mathrm{a}}$ & $264.9^{\mathrm{b}}$ \\
Peso promedio de muestras $(\mathrm{kg})$ & $6.54^{\mathrm{a}}$ & $3.04 \mathrm{~b}$ & $6.61^{\mathrm{a}}$ & $3.31^{\mathrm{b}}$ \\
Peso total de lote $(\mathrm{kg})$ & $32.712^{\mathrm{a}}$ & $15.217^{\mathrm{b}}$ & $33.055^{\mathrm{a}}$ & $16.556^{\mathrm{b}}$ \\
Rendimiento $(\mathrm{kg} / \mathrm{ha})$ & $65.406^{\mathrm{a}}$ & $30.434^{\mathrm{b}}$ & $66.110^{\mathrm{a}}$ & $33.113^{\mathrm{b}}$ \\
\hline
\end{tabular}

Letras distintas en la misma fila, los tratamientos son diferentes $(\mathrm{P}<0.05)$

Al considerar el rendimiento en forraje fresco de cada tratamiento, se aprecia que el $T_{3}$ (Asociación maíz-mucuna) y $T_{1}$ (maíz en monocultivo) fueron los de mayor rendimiento $(P<0.05)(66.110$ y $65.406 \mathrm{~kg} / \mathrm{ha})$, aunque $T_{1}$ fue superior $1.1 \%$ con respecto a $\mathrm{T}_{3}$, le siguieron con una diferencia del $49.9 \% \mathrm{~T}_{4}$ (Elefante con mucuna) y $\mathrm{T}_{2}$ con $53.9 \%$ (Elefante en monocultivo) con rendimientos de 33.113 y 30.434 $\mathrm{kg} / \mathrm{ha}$, respectivamente.

En los rendimientos de ensilaje por hectárea (Tabla 3), se aprecia que $T_{3}$ y $T_{1}$ fueron superiores $(\mathrm{P}<0.05), 61.181$ y $60.649 \mathrm{~kg} / \mathrm{ha}$, al compararlos con $\mathrm{T}_{4}$ y $\mathrm{T}_{2}$ : 
33.113 y $30.434 \mathrm{~kg} / \mathrm{ha}$ de forraje ensilado, respectivamente. $\mathrm{T}_{1}$ y $\mathrm{T}_{2}$ produjeron $49.27 \%$ más de ensilaje con relación a los otros dos tratamientos, de lo cual se deduce que el maíz es una buena alternativa para la alimentación de bovinos doble propósito, en la Altillanura Colombiana puesto que suministra gran cantidad de biomasa consumible, lo cual le provee energía para sus funciones productivas carne y leche, al adicionar mucuna, se hace el aporte de proteína y de esta forma se suple adecuadamente los requerimientos del ganado.

Tabla 3. Rendimiento de forraje ensilado por hectárea

\begin{tabular}{|c|c|c|c|c|}
\hline Tratamientos & $\mathbf{T}_{1}$ & $\mathbf{T}_{2}$ & $T_{3}$ & $\mathbf{T}_{4}$ \\
\hline \multirow{2}{*}{$\begin{array}{c}\text { Tamaño de cada muestra }\left(\mathrm{m}^{2}\right) \\
\text { Número de muestras por } \\
\text { tratamiento }\end{array}$} & 1.0 & 1.0 & 1.0 & 1.0 \\
\hline & 80 & 80 & 80 & 80 \\
\hline $\begin{array}{l}\text { Peso totalidad de muestras }(\mathrm{kg}) \\
\text { por tratamiento }\end{array}$ & $485,1^{a}$ & $240,4^{b}$ & $489,4^{a}$ & $242,6^{b}$ \\
\hline $\begin{array}{c}\text { Peso promedio de muestras }(\mathrm{kg}) \\
\text { Peso total de lote }(\mathrm{kg})\end{array}$ & $\begin{array}{c}6,06^{\mathrm{a}} \\
30.324^{\mathrm{a}}\end{array}$ & $\begin{array}{l}3,00^{b} \\
15.00^{b}\end{array}$ & $\begin{array}{c}6,11^{\mathrm{a}} \\
30.590^{\mathrm{a}}\end{array}$ & $\begin{array}{c}3,03^{\mathrm{b}} \\
15.015^{\mathrm{a}}\end{array}$ \\
\hline Rendimiento (kg/ha) & $60.649^{a}$ & $30.000^{b}$ & $61.18^{1 \mathrm{a}}$ & $30.030^{b}$ \\
\hline
\end{tabular}

Letras distintas en la misma fila los tratamientos son diferentes $(P<0.05)$

\section{Indicadores económicos}

Para determinar los indicadores económicos para cada tratamiento, se siguió un procedimiento que consistió en tomar como referencia la cotización de un producto de similares características del mercado local. Los costos determinados en el experimento se establecieron para la explotación de la hacienda Santa Martha, en la cual el ensilaje se produce para autoconsumo y por ello en su costo unitario se economiza el de las bolsas plásticas y de polipropileno, el empacado, la manipulación y transporte para un mercado, donde el rubro se calculó por cada $1.000 \mathrm{~kg}$ de ensilaje: en $\$ 46.700$ por tonelada. El valor del ensilaje en San Martín (Meta), tiene un valor de $\$ 160.000$, asumiendo que éste valor podría ser el posible precio de venta del ensilaje producido, Tabla 4.

En la rentabilidad económica del ensilaje por hectárea (Tabla 5), se aprecia que $T_{3}$ y $T_{1}$ fueron superiores $(P<0.05), 6.931 .807$ y $6.871 .532 \$ / h a$, al compararlos con 
T4 y T2: 3.402.399 y 3.402.399 \$/ha, respectivamente. T1 y T2 produjeron 49.27\% más de rentabilidad con relación a los otros dos tratamientos, de lo cual se deduce que el maíz aparte de ser una buena alternativa para la alimentación de bovinos doble propósito, en la Altillanura Colombiana, es una actividad agropecuaria que genera buen rendimiento económico.

Tabla 4. Costos de ensilaje, empacado y transporte por cada $1.000 \mathrm{~kg}$

\begin{tabular}{ccccc}
\hline Concepto & \multirow{2}{*}{ Unidad } & Cantidad & \multicolumn{2}{c}{ Valor } \\
& & & Unitario & Total \\
\hline Bolsa plástica, cal \# 7 & Bolsa & 31 & 400 & 12.400 \\
Bolsa polipropileno & Bolsa & 31 & 300 & 9.300 \\
Empacado & Jornal & 0.5 & 14.000 & 7.000 \\
Transporte y manipulación & Ton. & 1 & 18.000 & 18.000 \\
& Total (\$) & & & 46.700 \\
\hline
\end{tabular}

Tabla 5. Rendimiento económico del ensilado por tratamiento

\begin{tabular}{ccccc}
\hline Tratamientos & $\mathbf{T}_{\mathbf{1}}$ & $\mathbf{T}_{\mathbf{2}}$ & $\mathbf{T}_{\mathbf{3}}$ & $\mathbf{T}_{\mathbf{4}}$ \\
\hline $\begin{array}{c}\text { Rendimiento }(\mathrm{kg} / \mathrm{ha}) \\
\text { Costos de producción }(\$ / 1000\end{array}$ & $60.649^{\mathrm{a}}$ & $30.000^{\mathrm{b}}$ & $61.181^{\mathrm{a}}$ & $30.030^{\mathrm{b}}$ \\
$\mathrm{kg})$ & 46.700 & 46.700 & 46.700 & 46.700 \\
Valor de venta Ton $(\$)$ & 160.000 & 160.000 & 160.000 & 160.000 \\
Costos de producción total & $2.832 .308^{\mathrm{a}}$ & $1.401 .000^{\mathrm{b}}$ & $2.857 .153^{\mathrm{a}}$ & $1.402 .401^{\mathrm{b}}$ \\
(\$/1000 kg) & $9.703 .840^{\mathrm{a}}$ & $4.800 .000^{\mathrm{b}}$ & $9.788 .960^{\mathrm{a}}$ & $4.804 .800^{\mathrm{b}}$ \\
Valor total de Venta $(\$)$ & $6.871 .532^{\mathrm{a}}$ & $3.399 .000^{\mathrm{b}}$ & $6.931 .807^{\mathrm{a}}$ & $3.402 .399^{\mathrm{b}}$ \\
Beneficio / ha $(\$)$ & & &
\end{tabular}

Letras distintas en la misma fila los tratamientos son diferentes $(\mathrm{P}<0,05)$

\section{CONCLUSIONES}

En la variable agronómica rendimiento de biomasa en forraje fresco, los mejores resultados se presentaron en los tratamientos que involucraron al maíz en asociación con mucuna 61.181 Vs $60.649 \mathrm{~kg} / \mathrm{ha}$ para monocultivo, siendo el más bajo para elefante $30.000 \mathrm{~kg} / \mathrm{ha}$. Este resultado muestra dos aspectos: 1) La leguminosa asociada con la gramínea incrementa el rendimiento de forraje, y 2) el maíz en un solo corte es superior en producción al elefante.

El rendimiento del forraje ensilado presentó un comportamiento similar a la variable anterior, obteniéndose la mayor producción de biomasa: la asociación 
maíz-mucuna, y la menor con el pasto elefante, es de anotar que los tratamientos en los que hizo parte el maíz fueron los que presentaron los mejores resultados.

De acuerdo al análisis económico se deduce, que el mayor se obtuvo al ensilar la asociación de maíz-mucuna seguido de maíz en monocultivo, seguido de la asociación de pasto elefante y mucuna, y por último elefante en monocultuivo. Además el ensilaje de maíz asociado con mucuna presenta el mayor potencial para ser implementado en la producción de biomasa como estrategia de alimentación bovina.

Los cuatro tratamientos arrojaron valores de costos de producción de $\mathrm{kg}$ de biomasa ensilada inferiores a los que se encuentran en el mercado de suplementos comerciales, por ello para el ganadero es más rentable producir su propio ensilaje, que comprarlo, puesto que la asociación de maíz y mucuna, le ofrece una alternativa viable y económica.

\section{REFERENCIAS BIBLIOGRÁFICAS}

1. Alcaldía de San Martin. Plan de desarrollo Municipio de San Martín de los Llanos 2012-2015. 123 p. 2012.

2. Bernal EJ, Chaverra GH. El ensilaje en la alimentación de ganado vacuno. 1aㅡ edición. Tercer mundo editores. Bogotá, Colombia. 153 p. 2000.

3. Bertoia LM. Ensilaje de maíz: calidad técnica y biológica. Revista Angus, 250: 23-25. 2010.

4. Cárdenas JV, Sandoval CA, Solorio J. Composición química de ensilajes mixtos de gramíneas y especies arbóreas de Yucatán, México. Revista Técnica Pecuaria en México, 41 (3): 283-294. 2003.

5. Carranza J. Algunas técnicas para el análisis de resultados de investigación. Bogotá. 174 p. 1994.

6. Castillo M, Rojas A, WingChing R. Valor nutricional del ensilaje de maíz cultivado en asocio con Vigna (Vigna radiata). Agronomía Costarricense, 33 (1): 133-146. 2009.

7. Chacón PA, Vargas CF. Digestibilidad y calidad del Pennisetum purpureum cv. King grass a tres edades de rebrote. Agronomía meosoamerica, 20 (2): 339 408. 2009.

8. CORPOICA. Conservación y uso de soya forrajera para la alimentación de rumiantes en la Orinoquia Colombiana. Boletín técnico $N^{\circ} 37$. Villavicencio, Colombia. 20 p. 2003.

9. CORPOICA. Manejo agronómico de algunos cultivos forrajeros y técnicos para su conservación en la región Caribe Colombiana. Manual Técnico, Centro de Investigación Turipaná, Careté, Cordoba Colombia, 52 p. 2003. 
10. Cuartas CA, Naranjo JF, Murgueitio E. Desarrollo de un modelo silvopastoril intensivo con Leucaena leucocephala y pastos mejorados asociados a maderables de diferentes especies que fortalezcan la alianza entre la ganadería y la industria forestal en tres empresas ganaderas en el valle del río Cesar. Revista Colombiana de Ciencias Pecuarias, 22 (3): 515. 2009.

11. Garcés AM, Berrio L, Ruiz S, Serna JG, Builes AF. Ensilaje como fuente de alimentación para el ganado. Revista Lasallista de investigación, 1 (1): 66-71. 2004.

12. Jamioy D. Propuesta de indicadores de calidad edafológicos para valorar la influencia de los sistemas productivos sobre algunas propiedades físicas y químicas en suelos oxisoles del piedemonte llanero colombiano. Tesis de grado Magister en Ciencias Agrarias con énfasis en Suelos, Universidad Nacional de Colombia, Facultad de Ciencias Agropecuarias, 110 p. 2011.

13. Lemus LH., Lemus VE. Plantas de uso forrajero en el trópico cálido y templado de Colombia. Universidad de los Llanos. Villavicencio, Colombia. 360 p. 2004.

14.Zambrano C, Sánchez D, Uzcátegui J. Consumo y digestibilidad de pasto King grass (Pennisetum purpureum) fresco y ensilado con Leucaena leucocephala en ovinos en crecimiento. Revista Unellez, 24: 77-82. 2006. 